Keywords: MCMI-III, Exploratory and confirmatory factorial analysis, Personality disorders.

\title{
Exploratory and confirmatory factorial structure of the MCMI-III Personality Disorders: Overlapping versus non-overlapping scales
}

\author{
Lara Cuevas* \\ Luis F. García** \\ Antón Aluja*** \\ Óscar García**** \\ * Department of Social Psychology and \\ Methodology, Autonomous University of \\ Madrid \\ ** Department of Biological and Health \\ Psychology, Autonomous University of \\ Madrid \\ *** Department of Pedagogy and \\ Psychology, University of Lleida \\ **** Department of Pedagogy and \\ Psychology, European University of Madrid \\ SPAIN
}

\begin{abstract}
Background and Objectives: The aim of this study was to explore the factorial structure of the 14 Personality Disorder (PD's) scales of the MCMI-III for the overlapping and non-overlapping scales, independently. Previous exploratory studies using different factor extraction procedures inform that the structure of MCMI-III personality disorders has between 2 and 4 factors.

Methods: The present study used a large sample of 674 non-clinical subjects divided at random in two groups: a) calibration, and b) validation. In the calibration group, principal component analysis with orthogonal rotation was carried out, obtaining 2, 3 and 4 factors for the overlapping and non-overlapping scales independently. In the validation group, the three models were compared using confirmatory factorial analysis techniques.

Results and Conclusions: The exploratory and confirmatory results indicate that the 4factor solution is the most plausible. Although the congruence coefficients between nonoverlapping and overlapping scales in the 4-factor solution were higher, confirmatory factor analysis showed that models designed from overlapping scales did not fit well to data.
\end{abstract}




\section{Introduction}

The Millon Clinical Multiaxial Inventory ${ }^{1}$ is probably the most used self-report instrument for the assessment of the DSM-IV personality disorders ${ }^{2}$. During the past two decades, the original test has undergone two revisions. The Millon Clinical Multiaxial Inventory-Second Version ${ }^{3}$ was introduced in 1987 to coincide more accurately with the changes advanced in the Diagnostic and Statistical Manual of Mental Disorders, $3^{\text {rd }}$ edition revised ${ }^{4}$. Recently, the Millon Clinical Multiaxial Inventory-Third version ${ }^{5}$ was published to match the diagnostic guidelines advanced in DSM-IV ${ }^{6}$. Like the MCMI-II, the MCMI-III differs from its predecessor in important ways. Over half (95) of the original 175 items were replaced, and a new personality scale (Depressive) and a new clinical scale (Post-Traumatic Stress Disorder) were added. The itemweighting system was modified by reducing the weighting of prototypical items from the original three to two points, weighting the remaining items one point. The number of items on individual scales was reduced to minimize the statistical problems associated with excessive item overlap among scales.

Given these changes on the MCMI-III, research is needed to understand how well previous clinical and research findings with the MCMI-I and MCMI-II can be applied to the latest version. In the manual, Millon et $a l .{ }^{5}$ reported on cross-validation studies between the two instruments. However, factor analytic findings were not reported in the 1994 manual, and there was no correlation matrix between non-overlapping scales (only a correlation matrix between the full scales).

Factor analyses of earlier versions of the MCMI have produced somewhat inconsistent solutions ${ }^{7-11}$. Among the possible reasons for these inconclusive results are the following: a) Some studies have analysed the full overlapping scales, whereas others analysed the non-overlapping scales; b) many have conjointly factored the PD and clinical Axis-Itype scales of the MCMI, whereas others factored only the PD scales; c) some have factored items instead of scales; and d) some have used clinical samples, whereas others have used non-clinical samples.

Furthermore, differing criteria have been used to determine the number of factors to extract, which has also produced different solutions. Several researchers have explored the factorial structure of the MCMI with the aim of testing Millon's theory and how it is measured utilizing the instruments derived from it, whereas others have chosen a specific number of factors on the basis of previous empirical literature. However, it might be difficult to use the MCMI-III (or any prior edition) to conduct research on the theoretical model, as none of the scales within the MCMI-III correspond to the fundamental constructs of the model. There is no self-other, pleasure-pain, or active-passive scales within the MCMI-III, nor any scales to assess the circumplical emotionality and affiliation dimensions. In fact, its success as a clinical instrument is dependent primarily on its validity as a measure of the DSM-IV personality disorders, and most of the diagnostic criteria for the DSM-IV personality disorders were not based on Millon's ${ }^{12-14}$ theoretical model.

In one of the first studies, Retzlaff et al. ${ }^{15}$ explored the factor structure of the eight basic personality scales with five different participant samples and with a correlation matrix derived solely from the extent of the overlap among the scales. Support was obtained for a three-factor solution identified as Aloof-Social, Aggressive-Submissive, and Lability-Restraint. More recently, Dyce et al. ${ }^{16}$ conducted a factor analysis of 
the MCMI-III personality disorder scales, and compared the results with previous factor analyses. They indicated that the correlation matrices obtained with the $\mathrm{MCMI}^{1}$, the MCMI-II ${ }^{3}$, and the MCMI-III ${ }^{5}$ were reasonably consistent over time and settings: "The structure of these personality scales has remained the same across the (...) recent versions of the test" ${ }^{\prime 16}$ (p. 578).

$\mathrm{O}^{\prime} \mathrm{Connor}^{17}$ reanalyzed the factor structure of many personality disorder studies. They used four rules to decide how many factors should be extracted in each database: Parallel Analysis, Minimum average partial test, Standard error Scree test, and number of eigenvalues equal or greater than one. Of the 33 studies included, 12 administered a MCMI version. The four rules advise a number of factors between 2 and 4 depending on the study and the rule. Only two studies ${ }^{5,18}$ applied the third version of the MCMI, making them especially relevant to the present study. In both cases, the number of factors suggested was between 2 (Parallel Analysis, Minimum average partial test, and number of eigenvalues greater than one) and 3 (Standard error scree test). Also, it should be remarked that the number of factors advised by each rule was replicated in both studies.

However, Dyce et al. ${ }^{16}$ also indicated that a four-factor solution was the most consistent and compelling structural representation of the MCMI-III personality disorder scales, and that the four-factor solution was more consistent with the lexical five-factor model of personality ${ }^{19}$. The four-factor model is probably the most useful and relevant of the $\mathrm{n}$-factor patterns. The fourth dimension in this model is statistically weak, but it is necessary for providing a satisfactory representation of all PD's. The four-factor model is also consistent with Watson et al. ${ }^{20}$ claim that four dimensions are most useful in representing personality pathology, and it is the model that provides the most differentiation between PD's. However, the fourth dimension does not summarize enough covariation between PD's to meet the criteria for being a fullyfledged dimension. Only the ObsessiveCompulsive PD displayed a notable loading on this factor. The fourth dimension can therefore be viewed as a small singlet that exists orthogonally in the shadow of the three primary dimensions.

Finally, it is unknown whether the factorial structure is invariant for samples that are culturally and linguistically different from the original American standardization sample. For example, a European psychologist using a translated version of the MCMI-III cannot be sure that he or she is measuring the same traits as his or her counterpart in the US using the original version of the MCMI-III.

Note that only a few studies of the MCMIIII factor structure have been carried out. Furthermore, no study has ever been done in a non-English language. The aims of the present article were: (a) to test different two, three and four-factor solutions appearing in the literature, (b) to explore the role of the overlap among the scales on the factor structure, and (c) to use a confirmatory factor analysis approach to answer the question of what the best factor structure of the Spanish MCMI-III is.

\section{Method}

\section{Subjects}

Total sample comprised 674 subjects (37.8\% males and $62.2 \%$ women; in one case the sex was not informed). The average age was 33.19 (sd: 15.11) for males, and 31.10 (sd: 14.62) for females. Fifty per cent 
of the subjects were undergraduate university students from three Spanish universities (located at Barcelona, Madrid, and Lleida) and the remaining $50 \%$ were students' friends and relatives. Age frequency for the whole sample was as follows: 17-23 $(\mathrm{n}=$ $335 ; 49.9 \%) ; 24-29(\mathrm{n}=71 ; 10.6 \%) ; 30-44$ $(\mathrm{n}=69 ; 10.3 \%) ; 45-48(\mathrm{n}=58 ; 8.6 \%) ; 49-$ $53(\mathrm{n}=84 ; 12.5 \%) ; 54-79(\mathrm{n}=55 ; 8.2 \%)$.

\section{Measures}

Millon Clinical Multiaxial Inventory (MC MI-III). The MCMI-III ${ }^{5,21}$ is an inventory consisting of 175 true-false items from which scores on 14 Personality Disorders (PD's); 10 clinical syndrome scales can be computed. Additionally, the MCMI-III incorporates 3 "modifier" scales. Overlapping and non-overlapping were obtained by computing according to handbook instructions. Millon et al. ${ }^{5}$ designed the scales to explicitly align with the diagnostic criteria of the DMS-IV. Evidence for the validity of the English original version was provided in the form of correlations with ratings by clinicians, with collateral tests measuring identical constructs, and strong diagnostic efficiency statistics. The alpha coefficients reported in the test manual ranged from 0.67 to 0.89 and the test-retest values (5-14 days) ranged from 0.88 to 0.93 . As a Spanish version of the MCMI-III was not available when the present study was carried out, the MCMI-III was translated to Spanish by the authors of this study, under the supervision of the English Department of University of Lleida.

\section{Procedure and statistical analysis}

The MCMI-III was administered to psychology students in the classroom. Students were trained in the application of psychome- tric tests, and protocols were given to them to be administered to relatives and friends. Protocols were applied to subjects older than 25 for the purpose of obtaining a larger age distribution. According to the instruction in the MCMI-III manual ${ }^{21}$ profiles are considered valid if the total number of omitted or invalid responses (e.g., both a 'yes' response and a 'no' response to a single item) is less than 12 , if the validity index is less than 2 , and if the raw score on scale $X$ (disclosure) is within the range 34-178. Following this rules, all protocols could be considered valid and were processed statistically in the present sample.

Both overlapping and non-overlapping scores were computed. Therefore, all analyses were computed based on both kinds of scores. It is usually recommended to divide the sample when exploratory and confirmatory analyses are successively conducted for the same sample. Thus, the total sample was divided at random by selecting approximately $50 \%$ of the sample through the appropriate SPSS command. The number of subjects for both calibration and validation subsamples was 337 . The calibration sample was composed of 132 males $(39.2 \%)$ and 204 females $(60.5 \%)$ with a total average age of 31.91 (sd:15.19). The validation sample comprised 123 males (36.5\% males) and 213 females $(63.2 \%)$ with a total average age of 31.88 (sd: 14.48).

Exploratory factor analyses (EFA) were conducted through Principal Components (PC) with Varimax rotation analysis on the calibration sample. Number of factors was chosen previously since the present paper aimed to explore two, three and four-factor solutions used elsewhere. All statistical analyses were carried out with the SPSS 12.0.

Furthermore, a series of confirmatory factor analyses (CFA) of two, three, and four factors were performed to test which factor structure best fits the data for non-overlap- 
ping and overlapping scores separately on the validation sample. The most appropriate variance-covariances matrix was analysed with the Amos 5.0. statistical package. Successive models were designed following specifications of the Oblique Modest Loadings model ${ }^{22,23}$. In this model, all correlations between factors, and loadings larger than \pm 0.20 were set free. The Varimax solution of the calibration sample was considered as the criterion to decide what factor loadings were freely estimated. In order to identify the models, variances of latent exogenous variables (the factors and the error terms of the items) were fixed to 1 . The estimation of the parameters was computed using the Maximum Likelihood method. Maximum Likelihood (ML) has been the most frequently used CFA estimation method in research with personality and psychopathological questionnaires. Although Millon's personality disorders scales could show some deviances from the normal distribution, the ML method is robust when the assumption of normality is not severely violated ${ }^{24,25}$.
Studies using CFA have usually reported one or a few fit indices only. However, since no index is perfectly reliable, it is recommended that several fit indices should be used in conjunction to make a decision ${ }^{26}$. Also, when several models are compared, RMSEA and $\mathrm{ECVI}^{27}$ are of special importance because they include confidence intervals to make a decision on the grounds of statistical information $^{25}$. Also, RMSEA is one of the most appropriate practical choices when the Maximum Likelihood method is performed ${ }^{28}$.

\section{Results}

\section{Descriptives and alpha coefficients}

Table I shows the means, standard deviations, skewness, kurtosis and alpha coefficients of the MCMI-III non-overlapping and overlapping scales for the total sample. Alpha reliability coefficients were similar to the

Table I

Descriptive and internal consistency for non-overlapping and overlapping scales for the total sample.

\begin{tabular}{|c|c|c|c|c|c|c|c|c|c|c|}
\hline & \multicolumn{5}{|c|}{ Non-overlapping } & \multicolumn{5}{|c|}{ Overlapping } \\
\hline & M & SD & $\mathrm{S}$ & K & alpha & M & SD & $\mathrm{S}$ & K & alpha \\
\hline Schizoid & 2.58 & 2.84 & 1.36 & 1.83 & .67 & 4.63 & 3.74 & 1.26 & 1.71 & 68 \\
\hline Avoidant & 2.57 & 3.20 & 1.45 & 1.85 & .74 & 4.05 & 4.11 & 1.44 & 2.06 & .77 \\
\hline Dependent & 4.56 & 3.57 & .89 & .42 & .72 & 6.11 & 4.55 & 1.04 & .71 & .76 \\
\hline Histrionic & 8.14 & 4.00 & -.25 & -.96 & .71 & 16.29 & 4.90 & -.43 & -.39 & .74 \\
\hline Narcissistic & 2.77 & 2.85 & 1.21 & 1.36 & .58 & 13.76 & 3.98 & .35 & .35 & .64 \\
\hline Antisocial & 3.01 & 2.78 & .97 & .67 & .66 & 5.54 & 3.89 & .88 & .69 & .68 \\
\hline Sadistic & 3.21 & 3.10 & 1.02 & .52 & .73 & 6.04 & 4.66 & .91 & .39 & .74 \\
\hline Compulsive & 8.36 & 4.05 & -.02 & -.81 & .63 & 14.83 & 4.81 & -.25 & -.40 & .65 \\
\hline Passive-Aggressive & 5.69 & 3.55 & 68 & .11 & .72 & 7.51 & 4.83 & .74 & .16 & .76 \\
\hline Masochistic & 1.40 & 2.14 & 1.85 & 3.75 & .73 & 2.68 & 3.30 & 1.76 & 3.42 & .77 \\
\hline Schizotypal & 2.27 & 3.01 & 1.77 & 3.51 & .72 & 2.98 & 3.70 & 1.82 & 3.89 & .76 \\
\hline Borderline & 3.49 & 3.47 & 1.18 & 1.19 & .73 & 4.96 & 4.44 & 1.16 & 1.21 & .76 \\
\hline Paranoid & 3.53 & 3.69 & 1.14 & .79 & .74 & 4.45 & 4.57 & 1.33 & 1.67 & .79 \\
\hline Depressive & 3.20 & 3.71 & 1.33 & 1.36 & .82 & 4.08 & 4.72 & 1.49 & 2.00 & .84 \\
\hline
\end{tabular}

S: Skewness; K: Kurtosis 
original scales with ranges between 0.58 and 0.82 for non-overlapping scales and 0.64 and 0.84 for overlapping ones. Note that coefficients were similar between both kinds of scales, being somewhat lower for non-overlapping ones. Also, the skewness and kurtosis values demonstrate that scales did not violate severely the normality assumption, since no scale presented a skewness and kurtosis value higher than 2 and 7, respectively.

\section{Factor Analyses}

Factors were extracted from a Varimax principal components factor analysis including the 14 PD's scales. For non-overlapping scales, the Kaiser-Meyer-Olkin (KMO) measure of sampling adequacy was 0.879 , and Bartlett's Test of Sphericity (BTS) yielded an approximate Chi-Square of 1976.62 (df: $91 ; p<0.001$ ). The KMO measure of sampling adequacy is an index which examines the appropriateness of factor analysis. It should be 0.50 , and the higher the better. This measure of sampling adequacy compares magnitude of correlations with the magnitude of partial correlation coefficients. Small values indicate that correlations cannot be explained by other variables, and factor analysis may be inappropriate.

Table II shows the two, three and four -factor solutions for the non-overlapping scales. In the two-factor solution, most of the scales loaded on the first factor, suggesting a Neuroticism factor, whereas the second factor might be described as an Inhibition factor. In the three-factor solution, the latter was split into a factor formed by the Cluster B personality disorders and an Obsessive-Compulsive factor. The four-factor structure reproduces quite well the three factor solution plus an added factor mainly defined by the Histrionic scale (in negative). The percentage of variance accounted for in the 2, 3 and 4 solutions was 53.95, 61.98 and 69.15, respectively.

For overlapping scales, the Kaiser-MeyerOlkin (KMO) measure of sampling adequacy was 0.86, and Bartlett's Test of Sphericity (BTS) yielded an approximate Chi-Square of 3536.40 (df: $91 ; p<0.001$ ). Factor solutions for overlapping scales are presented in Table III. In the two-factor solution, almost all the

Table II

Factor solutions (2, 3 and 4 factors) obtained in non-overlapping scales.

Two-Factor

Three-Factor

Four-factor

\begin{tabular}{lccccccccc}
\hline Schizoid & $\mathbf{. 4 0 8}$ & $\mathbf{. 5 9 6}$ & $\mathbf{. 4 6 2}$ & -.012 & $\mathbf{. 5 9 7}$ & $\mathbf{. 3 3 3}$ & .288 & $\mathbf{. 3 4 9}$ & $\mathbf{. 5 9 8}$ \\
Avoidant & $\mathbf{. 6 2 9}$ & $\mathbf{. 5 3 5}$ & $\mathbf{. 7 9 8}$ & -.031 & .280 & $\mathbf{. 7 5 9}$ & .128 & .171 & $\mathbf{. 3 0 7}$ \\
Dependent & $\mathbf{. 5 2 9}$ & $\mathbf{. 3 6 3}$ & $\mathbf{. 6 8 5}$ & -.023 & .098 & $\mathbf{. 8 0 1}$ & -.082 & .232 & -.188 \\
Histrionic & .150 & $\mathbf{- . 6 3 5}$ & -.230 & $\mathbf{. 5 7 5}$ & -.275 & -.034 & .226 & .007 & $\mathbf{- . 9 0 0}$ \\
Narcissistic & $\mathbf{. 6 2 8}$ & -.279 & .195 & $\mathbf{. 7 4 5}$ & .155 & .126 & $\mathbf{. 7 9 1}$ & -.007 & -.068 \\
Antisocial & $\mathbf{. 5 2 4}$ &.- .551 & .230 & $\mathbf{. 6 1 6}$ &.- .395 & .152 & $\mathbf{. 5 6 9}$ & $\mathbf{- . 5 2 6}$ & -.152 \\
Sadistic & $\mathbf{. 6 5 7}$ & -.115 & .277 & $\mathbf{. 6 7 1}$ & .281 & .211 & $\mathbf{. 7 5 3}$ & .115 & .005 \\
Compulsive & .125 & $\mathbf{. 5 7 6}$ & .050 & .014 & $\mathbf{. 8 4 6}$ & .125 & .113 & $\mathbf{. 8 9 5}$ & .056 \\
Passive-Aggressive & $\mathbf{. 7 7 1}$ & .026 & $\mathbf{. 6 2 7}$ & $\mathbf{. 4 4 6}$ & .065 & $\mathbf{. 5 8 3}$ & $\mathbf{. 5 0 4}$ & -.055 & .022 \\
Masochistic & $\mathbf{. 6 9 6}$ & .241 & $\mathbf{. 7 2 9}$ & .193 & .089 & $\mathbf{. 6 7 9}$ & .295 & -.033 & .172 \\
Schizotypal & $\mathbf{. 7 7 1}$ & .183 & $\mathbf{. 7 2 2}$ & $\mathbf{. 3 1 7}$ & .122 & $\mathbf{. 6 6 8}$ & $\mathbf{. 4 1 7}$ & -.014 & .136 \\
Borderline & $\mathbf{. 7 8 3}$ & -.074 & $\mathbf{. 6 9 8}$ & $\mathbf{. 4 0 9}$ & -.163 & $\mathbf{. 6 8 7}$ & $\mathbf{. 3 9 1}$ & -.222 & -.110 \\
Paranoid & $\mathbf{. 7 3 2}$ & .231 & $\mathbf{. 5 3 1}$ & $\mathbf{. 4 4 9}$ & $\mathbf{. 4 3 6}$ & $\mathbf{. 4 9 6}$ & $\mathbf{. 5 6 9}$ & $\mathbf{. 3 0 7}$ & .098 \\
Depressive & $\mathbf{. 7 2 6}$ & $\mathbf{. 4 1 0}$ & $\mathbf{. 8 5 3}$ & .074 & .156 & $\mathbf{. 8 4 0}$ & .171 & .085 & .160 \\
\hline
\end{tabular}

(a) Loadings larger than \pm .30 are in boldface. 
scales loaded on the first factor with the exception of the Narcissistic and ObsessiveCompulsive scales. The second factor is mainly defined by the scales grouped on Cluster B, and (in negative) the Avoidant and ObsessiveCompulsive scales. In the three-factor solution, the second factor was formed by the Antisocial and Obsessive-Compulsive scales, and the third one by the Narcissistic. The fourfactor solution shows a "Neuroticism" factor again, an "Introversion" one with Narcissistic and Schizoid scales loading largely (but in the opposite direction) on this second factor, whereas the third and fourth ones were mainly depicted by the Narcissistic and Obsessive-Compulsive scales, respectively. The percentage of variance accounted for in the 2, 3 and 4 solutions was 66.83, 74.96 and 81.99 , respectively. Finally, congruence coefficients between non-overlapping and overlapping scales in the four-factor solution were computed. All values were higher than 0.90 .

Table IV shows the results of the models for non-overlapping and overlapping scales.

Table III

Factor solutions ( 3 and 4 factors) obtained in overlapping scales.

\begin{tabular}{lrrrrrrrrr} 
& \multicolumn{3}{c}{ Two-Factor } & \multicolumn{3}{c}{ Three-Factor } & \multicolumn{3}{c}{ Four-factor } \\
\hline Schizoid & $\mathbf{. 6 9 8}$ & -.283 & $\mathbf{. 7 2 3}$ & .003 & $\mathbf{- . 3 7 9}$ & $\mathbf{. 3 3 6}$ & .197 & -.070 & $\mathbf{. 8 1 8}$ \\
Avoidant & $\mathbf{. 8 5 5}$ & $\mathbf{- . 3 0 9}$ & $\mathbf{. 8 5 8}$ & -.273 & -.135 & $\mathbf{. 6 9 1}$ & -.087 & -.038 & $\mathbf{. 5 8 9}$ \\
Dependent & $\mathbf{. 7 3 7}$ & -.066 & $\mathbf{. 7 2 1}$ & -.250 & .180 & $\mathbf{. 8 8 4}$ & -.146 & -.044 & .007 \\
Histrionic & $\mathbf{- . 4 8 2}$ & $\mathbf{. 6 2 7}$ & $\mathbf{- . 4 9 6}$ & $\mathbf{. 4 4 7}$ & $\mathbf{. 4 2 3}$ & -.121 & .274 & .069 & $\mathbf{. . 9 0 8}$ \\
Narcissistic & -.242 & $\mathbf{. 7 1 9}$ & -.225 & $\mathbf{. 8 9 1}$ & .120 & -.248 & $\mathbf{. 8 1 3}$ & .111 & $\mathbf{- . 3 5 6}$ \\
Antisocial & $\mathbf{. 3 5 9}$ & $\mathbf{. 7 7 9}$ & $\mathbf{. 3 3 3}$ & $\mathbf{. 4 5 6}$ & $\mathbf{. 6 5 6}$ & .237 & $\mathbf{. 4 5 8}$ & $\mathbf{. 7 6 0}$ & .000 \\
Sadistic & $\mathbf{. 5 9 3}$ & $\mathbf{. 5 7 5}$ & $\mathbf{. 5 9 6}$ & $\mathbf{. 5 8 8}$ & .247 & $\mathbf{. 4 5 4}$ & $\mathbf{. 6 6 6}$ & $\mathbf{. 3 1 8}$ & .135 \\
Compulsive & -.033 & $\mathbf{- . 6 4 6}$ & .018 & -.046 & $\mathbf{- . 8 6 5}$ & .006 & .026 & $\mathbf{. . 9 4 5}$ & .131 \\
Passive-Aggressive & $\mathbf{. 8 0 1}$ & $\mathbf{. 3 5 2}$ & $\mathbf{. 7 9 2}$ & .241 & .283 & $\mathbf{. 7 2 4}$ & $\mathbf{. 3 5 6}$ & .282 & .194 \\
Masochistic & $\mathbf{. 8 6 6}$ & .014 & $\mathbf{. 8 5 7}$ & -.088 & .136 & $\mathbf{. 8 1 9}$ & .057 & .104 & .298 \\
Schizotypal & $\mathbf{. 8 4 1}$ & .060 & $\mathbf{. 8 4 2}$ & .067 & .047 & $\mathbf{. 7 3 0}$ & .219 & .076 & $\mathbf{. 3 6 1}$ \\
Borderline & $\mathbf{. 7 4 8}$ & $\mathbf{. 4 5 4}$ & $\mathbf{. 7 2 2}$ & .147 & $\mathbf{. 5 1 8}$ & $\mathbf{. 7 6 7}$ & .224 & $\mathbf{. 4 3 1}$ & .011 \\
Paranoid & $\mathbf{. 7 7 2}$ & .143 & $\mathbf{. 7 9 3}$ & $\mathbf{. 3 8 1}$ & -.150 & $\mathbf{. 6 2 9}$ & $\mathbf{. 5 3 3}$ & -.104 & $\mathbf{. 3 3 6}$ \\
Depressive & $\mathbf{. 8 7 5}$ & -.064 & $\mathbf{. 8 6 6}$ & -.168 & .107 & $\mathbf{. 8 6 4}$ & -.020 & .036 & .276 \\
\hline
\end{tabular}

(a) Loadings larger than \pm 0.30 are in boldface.

Table IV

Fit indexes ${ }^{(\mathrm{a})}$ of the analysed models.

\begin{tabular}{|c|c|c|c|c|c|c|c|c|c|}
\hline \multirow{2}{*}{ Models } & \multicolumn{7}{|c|}{ Non-overlapping scales } & \multirow[b]{2}{*}{ RMSEA } & \multirow[b]{2}{*}{ ECVI } \\
\hline & $\chi^{2(b)}$ & df & $\chi^{2} / \mathrm{df}$ & GFI & AGFI & NFI & CFI & & \\
\hline Two-factor & 315.48 & 71 & 4.44 & .87 & .81 & .84 & .87 & .11 & 1.22 \\
\hline Three-factor & 224.42 & 67 & 3.35 & .91 & .86 & .89 & .92 & .09 & 96 \\
\hline Four-factor & 177.22 & 62 & 2.86 & .93 & .88 & .91 & .94 & .08 & .84 \\
\hline \multicolumn{10}{|c|}{ Overlapping scales } \\
\hline Two-factor & 742.82 & 70 & 10.61 & .75 & .62 & .79 & .81 & .18 & 2.59 \\
\hline Three-factor & 731.29 & 66 & 11.08 & .76 & .62 & .80 & .81 & .18 & 2.58 \\
\hline Four-factor & 457.71 & 60 & 7.63 & .83 & .70 & .87 & .89 & .15 & 1.74 \\
\hline
\end{tabular}

(a) df: Degrees of Freedom. GFI: Goodness of Fit Index. AGFI: Adjusted Goodness of Fit Index. NFI: Normed Fit Index. CFI: Comparative Fit Index. RMSEA: Root Mean Square Error of Approximation. ECVI: Expected Cross-Validation Index.

(b) The associated $\mathrm{p}$ values were always lower than 0.001 . 
As can be seen, overlapping models were always worse than non-overlapping. Also, two and three- factor models did not fit well to data, irrespective of the sort of scale. The four-factor model for the non-overlapping scales presented a good fit, with acceptable values for the $\chi^{2} / \mathrm{df}$, GFI, NFI, CFI, and RMSEA $^{29}$. Also, it reached the lowest value for the ECVI fit index.

\section{Discussion}

The main aim of the present study was to analyze the factor structure of the Spanish version of the MCMI-III. Given that MCMI provides one particular view on the structure of PDs, it is important to examine the nature of this view more carefully. Rules for determining the number of factors to be extracted have produced differing results in the literature. For this reason, a more interesting approach is to report different solutions of two, three, and four factors, as in Dyce et al. ${ }^{16}$

All the solutions in our data suggest that a degree of integration of the different structures can be achieved. The first two factors were highly similar across all factor solutions. This was especially so in the case of the first, which included almost all scales and thus indicated a pathological factor related to emotional liability. A third factor appeared in both three and four factor solutions. Thus, it is fair to state that there was a degree of stability across the various solutions in the present study.

Previous factor solutions in the literature align reasonably well with the various solutions in Tables II and III. The problem with extracting fewer factors is that the 14 PD scales lump together and their distinctive- ness is lost. Although this will almost always occur in factor analytic attempts to simplify complex relations, the problem is perhaps too severe for the two and threefactor solutions to be useful. For example, extracting three factors permitted compulsive disorder to emerge from having no role in the two-factor solution and extracting four factors provided more differentiation of the Schizoid, Avoidant and Histrionic PDs from scales with which they were previously clustered. The four-factor solution thus best achieved the goal of simplification without excessive lost of information. Watson et al..$^{20}$ also claimed that PD's can be conceptualized in terms of four higher order factors. Further studies ${ }^{17}$ also supported a factor structure of personality disorders composed of four factors, the fourth being composed exclusively of the ObsessiveCompulsive disorder.

Quite a few studies have indicated that the DSM-IV personality disorders are readily understood as maladaptive variants of the domains and facets of the FFM, identifying many of these four broad factors of personality disorder symptoms identified by Livesley et al..$^{30}$ as emotional dysregulation, dissocial, inhibitedness, and compulsivity. These four broad domains align well with four of the five domains of the FFM (i.e. neuroticism, agreeableness, introversion, and conscientiousness, respectively). Therefore, this study supposes another piece of evidence favouring a model of personality disorders based on dimensional personality models. One such proposal has been already developed $^{31}$, as well as the necessary instruments to apply personality traits in clinical contexts $^{32}$.

Finally, one no less important contribution of the present paper is to reveal the possible role of the overlap among scales. The MCMI is a popular but sometimes contro- 
versial measure of PD's due to the fact that some items are used in computing scores for several scales. Psychometric properties were highly similar between non-overlapping and overlapping scales, alpha coefficients being almost equal. Also, skewness and kurtosis did not show sharp differences. Since inter-scales correlations are higher for overlapping scales, it is not surprising that loadings and percentage of variance were larger for this kind of solution. This did not, however, result in a marked difference with non-overlapping solutions. Note that the same factors emerge in the different solutions, with minimal differences on specific loadings. Also, it is remarkable that congruence coefficients between non-overlapping and overlapping scales in the four-factor solution were higher than 0.90 .

In spite of these initial similarities between overlapping and non-overlapping scales, results of confirmatory factor analysis showed that models designed from overlapping scales did not fit well to data. It should be noted that the four-factor model for overlapping scales fitted worse than the two-factor model for non-overlapping. It is also noteworthy that this worse fit was produced in spite of the higher percentage of variance accounted for when the overlapping scales were factor analyzed. This was not entirely unexpected, however, taking into account the particular scale construction using single items on multiple primary scales, the brevity of each item pool, and the high covariance expected due to the polythetic nature of the theory ${ }^{33}$, which probably make, as other researchers considering CFA in analyzing Millon instrument have noted ${ }^{34}$, CFA inappropriate for applications involving the MCMI-III. It is our view, however, that such methodology can be useful in making clear which the best structure in both overlapping and non-overlapping scales is.
In regard to this point, confirmatory factor analysis reinforces the conclusions obtained for exploratory factor analysis, since the four factor model for non-overlapping scales presented a good fit to data. Two and three-factor models obtained bad fit indices, which lend support to the conclusion about the oversimplification of the two and three-factor solutions mentioned above. Finally, the non-clinical nature of the sample is a major limitation of the present study. Future studies should test if these conclusions may be applied to clinical populations.

\section{References}

1. Millon T. Millon Clinical Multiaxial Inventory (MCMI) Manual. Minneapolis MN: National Computer Systems; 1977.

2. Watkins CE, Campbell VL, Nieberding R, Hallmark R. Contemporary practice of psychological assessment by clinical psychologists. Prof Psychol-Res Pr 1995; 26: 54-60.

3. Millon T. Manual for the MCMI-II 2nd ed. Minneapolis MN: National Computer Systems; 1987.

4. American Psychiatric Association. Diagnostic and Statistical Manual of Mental Disorders. Third Edition, Revised. Washington, DC; 1987.

5. Millon T, Millon C, Davis R. Millon Clinical InventoryIII. Minneapolis MN: National Computer Systems; 1994.

6. American, Psychiatric Association. Diagnostic and statistical manual of mental disorders. 4th ed. Washington, DC: American Psychiatric Association; 1994.

7. Choca JP, Petersen CA, Shanley LA. Factor analysis of the Millon Clinical Multiaxial Inventory. J Consult Clin Psych 1986; 54: 253-255.

8. Helmes E. Stability in the internal structure of the Millon Clinical Multiaxial Inventory. Psychopathol Behav 1989; 11: 327-338.

9. Lorr M, Retzlaff PD, Tarr HC. An analysis of the MCMI-I at the item level. J Clin Psychol 1989; 45(6): 884890.

10. Lorr M, Strack S, Campbell L, Lamnin A. Personality and symptom dimensions of the MCMI-II: an item factor analysis. J Clin Psychol 1990; 46(6): 749-754. 
11. McCann JT. Convergent and discriminative validity of the MCMI-II and MMPI personality disorder scales. Psychol Assess 1991; 3: 9-18.

12. Millon T. Modern psychopathology: A biosocial approach to maladaptive learning and functioning. Philadelphia: Saunders; 1969.

13. Millon T. Disorders of personality: DSM-III Axis II. New York: Wiley; 1981.

14. Millon T, Davis RD. Disorders of personality: DSMIV and beyond. New York: Wiley; 1996.

15. Retzlaff PD, Gibertini M. Factor structure of the MCMI basic personality scales and common-item artifact. J Pers Assess 1987; 51(4): 588-894.

16. Dyce JA, O’Connor BP, Parkins SY, Janzen HL. Correlational structure of the MCMI-III personality disorder scales and comparisons with other data sets. J Pers Assess 1997; 69(3): 568-582.

17. O'Connor BP. A search for consensus on the dimensional structure of personality disorders. J Clin Psychol 2005; 61(3): 323-345.

18. O'Connor BP, Dyce JA. A test of models of personality disorder configuration. J Abnorm Psychol 1998; 107(1): 3-16.

19. Costa PT, Widiger TA. Personality disorders and the five-factor model of personality. Washington DC: American Psychological Association; 2002.

20. Watson D, Clark LA, Harkness AR. Structures of personality and their relevance to psychopathology. J Abnorm Psychol 1994; 103(1): 18-31.

21. Millon T, Davis R, Millon C, Grossman S. MCMI-III manual. 3rd. Minneapolis, MN: Pearson Assessments; 2006.

22. Aluja A, García O, García LF, Seisdedos N. Invariance of the "NEO-PI-R" factor structure across exploratory and confirmatory factor analyses. Pers Individ Dif 2005; 38: 1879-1890.

23. McCrae RR, Zonderman AB, Costa PT, Bond MH, Paunonen SV. Evaluating replicability of factors in the Revised NEO Personality Inventory: Confirmatory Factor Analysis Versus Procrustes Rotation. J Pers Soc Psychol 1996; 70: 552-566.

24. Bollen KA. Structural equations with latent variables. New York: Wiley; 1989.

25. Fabrigar LR, Wegener DT, MacCallum RC, Strahan EJ. Evaluating the use of exploratory factor analysis in psychological research. Psychol Methods 1999; 4: 272-299.
26. Sharma S, Mukherjee S, Kumar A, Dillon WR. A simulation study to investigate the use of cutoff values for assessing model fit in covariance structure models. J Bus Res 2005; 58: 935-943.

27. Browne MW, Cudeck R. Alternative ways of assessing model fit. In: Bollen KA, Long JS, eds. Testing structural equation models. Newbury Park CA: Sage; 1993. p. 136-162.

28. Sugawara HM, MacCallum RC. Effect of estimation method on Incremental fit indexes for covariance structure models. Applied Psychol Meas 1993; 17: 365-377.

29. Hu L, Bentler PM. Cutoff criteria for fit indexes in covariance structure analysis: Conventional criteria versus new alternatives. Struct Eqn Model 1999; 6: 1-55.

30. Livesley WJ, Jackson DN, Schroeder ML. A study of the factorial structure of personality pathology. J Pers Disord 1989; 3: 292-306.

31. Costa PT, McCrae RR. A Five-Factor Model perspective on personality disorders. In: Strack S, ed. Handbook of personology and psychopathology psychopathology: Essays in honor of Theodore Millon. New York: Wiley; 2005.

32. Trull TJ, Widiger TA. The Structured Interview for the Five Factor Model of Personality. In: De Raad B, Perugini M, eds. Big five assessment. Bern, Switzerland: Hogrefe Huber; 2002. p. 147-170.

33. Choca J. Review of the Millon Index of Personality Styles. In: Impara JC, Plake BS, eds. The thirteenth mental measurements yearbook. Lincoln NE: Buros Institute of Mental Measurements; 1998.

34. Derksen J, Sloore H. Issues in the international use of psychological tests. In: Strack S, ed. Handbook of personology and psychopathology psychopathology: Essays in honor of Theodore Millon. New York: Wiley; 2005.

Address for correspondence:

Lara Cuevas

Department of Social Psychology and Methodology,

Autonomous University of Madrid.

C/ Ivan Pavlov, 6

28049 Madrid (Spain).

Phone: + 914975182 .

e-mail: lara.cuevas@uam.es 